CuPAUAM. 18-1991, 179-188

\title{
EL CABALLO DE BRONCE DE CANCHO ROANO *
}

\author{
Sebastian Celestino Perez. \\ JOSE MANUEL JULian RODRIGUEZ
}

\section{Resumen}

Este trabajo da a conocer una pieza excepcional hallada en Cancho Roano durante la campana de excavaciones de 1990 . Se trata de un caballo de bronce de pequeño tamaño y muy buena factura, que quizá formó parte de un carrito votivo. Formaba parte de un depósito vorivo en la esrancia W-2, una de las habitaciones contiguas al edificio principal.

\section{Summary}

This paper presents an exceptional find discovered at the size of Cancho Roano during the 1990 field season. It is a bronze horse of small size and very good craftmanship, perhaps part of a votive chariot. The horse was found as part of a deposit of votive nature, found in Room W-2, one of the departments adjacent to the main building.

El presente trabajo sólo pretende dar a conocer una pieza excepcional aparecida en el yacimiento protohistórico de Cancho Roano, en Zalamea de la Serena; se trata de un caballo, o más bien una yegua, de bronce aparecido en el transcurso de la campaña de excavación llevada a cabo en 1990. La importancia de la escultura nos obliga a su rápida divulgación para el conocimiento de los especialistas; no obstante, volveremos a acometer su estudio una vez lista la memoria del sector occidental del yacimiento, en una de cuyas estancias fue hallado.

* El caballo apareció en la campafía de excavación realizada bajo el mecenazo de D. Barrolomé Gil Sanzacruz, a quien nunca podremos agradecer su enzusiasno y desinzeresada atención hacia zodo lo relacionado con el yacimienzo de Cancho Roano. También quezenos agradecer a Guillerno Kurra, Dizecror del museo de Badajoz, las facilidades preseadas; a Mazla Jerís Castellanos por su celeridad en la rescauración de la pieza; y a Salvador Rovira, por la realización de los análisis mecalográncos y su ayuda 
Tras el toral descubrimiento del edificio de Cancho Roano (1), a partir de 1987 nos planteamos la excavación de sus alrededores, los cuales han deparado nuevas estructuras arquitectónicas e ingentes cantidades de material íntimamente ligados al Palacio-Santuario (2).

El edificio se encuentra rodeado por una sucesión de estancias contiguas que sólo se interrumpen en el lado oriental, coincidiendo con el patio, para dejar diáfano el acceso al monumento. Salvo en esta zona oriental donde se han hallado cuatro estancias organizadas dos a dos a ambos lados del patio, el resto de los lados está compuesto por seis estancias cada uno, de los que se han excavado todos los del Norte, cuatro del Oeste y tan sólo uno del Sur. Estos espacios se organizan a partir de un muro que corre paralelo a la terraza del edificio, separado de este por casi $2 \mathrm{mts}$. a modo de pasillo. Todas las estancias tienen un ancho de 2 mts. mientras que su largo es variable, oscilando en torno a los 3,50 mts. El lado exterior se encuentra completamente cerrado por un muro de adobe, por lo que las puertas se comunican con el pasillo, siendo el patio oriental la salida común para todas estas estancias.

A partir del hallazgo in situ del depósito de material de la habitación N-6 (3), hemos venido considerando como ofrendas al Santuario todos los conjuntos encontrados, la mayoria de ellos muy alterados por la cremación intencionada del lugar, pero algunos muy bien conservados, como el mencionado de la zona Norte o dos de las estancias de la zona Oeste. En una de estas, la denominada W-2, aún por excavar en su totalidad por la presencia de una encina que ocupa parte del espacio, se halló in situ un depósito de material consistente en varios platitos votivos de los denominados de margarita (4), un skyphos ático, un telar carbonizado con su juego de pesas completo, asi como diversos elementos de adorno entre los que destaca un escarabeo de lidita. Una gran ánfora y otros vasos de menor tamaño completaban el depósito. En uno de los lados y sobre una pequeña banqueta de adobe se halló un pequeño depósito consistente en una olla, un plato, una pesa de telar y el caballo de bronce tumbado; junto a la banqueta se asentaba el ánfora mencionada y una placa de bronce con un cervatillo de similares características a las aparecidas en el interior del edificio, si bien estas presentan siempre figuras de équidos (5).

Descripción de la pieza.- Se trata de un bronce, con un peso de 1.351 grs., que representa un équido enjaezado. Las medidas son: $22 \mathrm{cms}$. de largo y $15 \mathrm{cms}$. de altura. (Fig. 1)

Ha sido moldeado en dos partes; la anterior comprende la cabeza, cuello y cuartos delanteros hasta la cruz, y la posterior, la grupa, cuartos traseros y cola. La parte anterior, mediante un cuerpo cilíndrico, rebajado y hueco, se introduce en la posterior quedando ambas unidas por tres rema-

(1) La bibliografia sobre el yacimiento de Cancho Roano es nuy extensa, por llo nos limitaremos a dar referencias de los trabajos nás generales sobre el yacimiento: Maluquer de Motes, J. 1981 y 1983. El Santuario Protobistárica de Zalamea de La Serena (Badajoz). P.I.P. IV y V Barcelona, Maluq̨uer de Motes, J.; Celestino, S.; Gracia, F.; Mutillla, G. 1986. El Santuario Procohiszórico de Zalamea de la Serena (Badajoz). P.I.P. XIV Bazcelona. Maluquer de Mozes, J. ; Gracia, F.; Munilla, G.; Celestino, S. 1987. Cancho Roano. "Un Palacio-Santuario del siglo V a.C." Revirza de Arqueologia 74. Una nueva visión del edificio principal en AlmagroGorbea, M.; Dominguez, A.; López-Ambice, F. e.p. "Cancho Roano. Un palacio orientalizante en la Peninsula iberica" Madrider Mitreilungen 3 I (1990). Y una sintesis de conjunto con las últimas excavaciones zealizadas en CELESTINO, S.. e.p. "Cancho Roano. Un centro comercial de carácter político-religioso e influencia orienzal" Revista di Studi FeniciXVIII, 1

(2) Celesino, 5. e.p. "El yacimiento de Cancho Roano. 1986-1990" Extremadura Arquealógica II. Cáceres 1991.

(3) Celesrino, S.i Jiménez, F.j. 1989. "Una ofrenda en la ešancia N-á del Palacio-Santuario de Cancho Roano" Archivo Español de Arqueologia 62.pp. 226-235.

(4) Maluquer de Motes, J. 1981, pp. 87-90.

(5) Maltuquer de Motes, J. 1983; fig. 24 y 25. 
ches, existiendo además dos perforaciones cilíndricas, una sobre la cruz y otra en la parte derecha media, sobre la manta del animal, y un remache que sobresale algo más de un centímetro en la parte izquierda de la manta, que no parece corresponder a la función de unir ambas partes.

$\mathrm{El}$ animal, al paso, tiene la mano y pata izquierda adelantadas y las derechas atrasadas. A pesar de ser una figura naturalista, no exenta de un cierto hieratismo, no ofrece un estudio anatómico detallado, limitándose el artesano a resolver la escultura con una buena plasmación de los volúmenes, que los presenta lisos salvo el detalle de la incisión, muy realista, que subraya los corvejones.

Tampoco aparece representado el sexo del animal, por lo que atendiendo al naturalismo general de la pieza, supondremos que se trata de una yegua.

Presenta ésta la cerviz recta y estirada, con el cuello, de sección ligeramente romboidal, ancho en la base y estrecho en la unión con la cabeza, algo baja. El maxilar inferior sobresale del cuello, con la curva inferior bien marcada.

Los ojos son almendrados y saltones, con los párpados marcados por un leve resalte y el globo ocular abombado y saliente.

Dos rehundimientos de forma alargada indican los ollares. Una incisión angular marca la boca, presentando los labios inferior y superior un ligero relieve.

Las orejas, triangulares, están abatidas hacia las crines, con los huecos de los oídos laterales.

Las crines están peinadas en guedejas, lisas, muy bien diferenciadas, todas peinadas hacia el lado izquierdo. Estas caen rectas sobre el cuello acabando en punta. Se extienden desde detrás de la oreja hasta la cruz.

La grupa es recta y el vientre presenta una convexidad natural. La cola, lisa, arranca recta, curvándose enseguida para bajar formando un ángulo de noventa grados, disminuyendo poco de grosor, para acabar estrecha y algo puntiaguda, un poco por encima de los espolones traseros.

Las patas están bien modeladas; las delanteras señalan ligeramente las rodillas mediante un pequeño ensanchamiento y presentan más marcadamente el espolón. Las patas traseras señalan, también con claridad, el menudillo del espolón y los corvejones. Los cascos están bien diferenciados.

De los cascos de las patas delantera derecha e izquierda trasera salen dos pestañas, de sección rectangular, perforadas, conservando aún una de ellas, la trasera izquierda, un pequeño clavo, también de bronce, doblado, que serviría la pieza sobre una plataforma que ha desaparecido.

Schulten (6) recoge algunas citas de autores clásicos sobre los caballos de la Península Ibérica que ilustran muy bien la pose de esta figura. Por ejemplo, Plinio relata de los asturcones que "los caballos no tienen la manera habitual de correr sino más bien un trote suave, que logran alargando las piernas alternativamente (es decir, moviendo como el camello, alternativamente las dos piernas izquierdas y las dos derechas), ..." y Silio añade que "... acelera los pasos (al correr), con la cerviz tiesa...". Hay que decir sin embargo que las opiniones de los geógrafos clásicos sobre los caballos hispanos no son unánimes y, en algunos casos son, incluso, contrapuestas.

\section{DESCRIPCION DEL ATALAJE}

Da la impresión de que ha sido representado de una forma algo negligente, al no figurar algunos elementos necesarios de los arreos.

(6) Schulten, A. 1963. Geografia y emografia antiguas de la Peninsula lberica. Vol. II. C.S.I.C. Madzid. pp. 479-499. 
Respecto al aparejo de cabeza observamos cinco piezas: en primer lugar destaca la visera, de buen tamaño, sobre la frente del animal, con la forma del país de un abanico desplegado. Es de sección rectangular y sus superficies, tanto la inferior como la superior, son lisas, sin ningún tipo de decoración.

Un segundo elemento es el guardanucas, estrecho y alargado, también liso, que parte claramente del centro de la superficie superior de la visera, siendo algo indefinida su terminación sobre las crines, una vez sobrepasadas las orejas.

La tetcera y cuarta piezas, gemelas, colocadas cada una a un lado de la cabeza del animal, descienden rígidamente desde los lados de la visera hasta llegar casi a los belfos, uniéndose por el exterior a los extremos de las riendas. Estas piezas, por su curvatura y forma, con una progresiva disminución de grosor hasta acabar en una punta algo roma, parecen representar unos cuernos de bóvido.

Finalmente, la rienda, sencilla, con los extremos entre la boca del animal y la parte interior de las dos piezas gemelas anteriormente descritas. Es bastante gruesa y descansa libre sobre el cuello del équido, cerca ya de la cruz.

Hemos dicho al comenzar la descripción del atalaje que parecian faltar algunos elementos necesarios. Podemos considerar que la visera, además de ser un objeto de adorno y, a la vez, de protección, podía ejercer la misma función que la frontalera y, del mismo modo, las dos piezas laterales, es decir, los cuernos de bóvido podían servir, como la visera, de adorno y protección y también sustituir en su función a los montantes.

Sin embargo no están representadas de ninguna forma ni testera ni ahogadero, elementos de sujección necesarios en la cabezada y, en cuanto a las alas del bocado, nada se puede decir pues tampoco aparecen representadas.

Referente a la montura, se observan con claridad el petral y la manta o cobertura. El petral, sencillo, decorado con un sogueado, se desliza, paralelo a la rienda, desde la parte inferior del cuello hasta unirse, casi en la cruz, con la parte anterior de la manta, desapareciendo entonces el sogueado y continuando como un reborde liso que contornea la cobertura, la cual presenta sus cuatro esquinas estiradas con las puntas redondeadas. Este reborde, de sección rectangular, desaparece en algunas zonas, bien porque no ha sido modelado, como entre la cruz y la grupa, o bien, porque ha sido limado, como puede apreciarse en la parte superior, sobre la grupa, y a continuación de las puntas delanteras derecha e izquierda de la manta.

La cincha que sujeta la cobertura no se representa claramente; sin embargo, justo en la zona de unión de la parte posterior de las dos que forman el animal, aparece apuntado el comienzo de lo que tal vez fuera la cincha, subiendo el reborde de la cobertura a lo largo de la línea de unión de las dos partes, aunque se interrumpe y desaparece enseguida a la altura del remache saliente situado en el lado izquierdo del animal y un poco más arriba, aunque con el reborde menos saliente, en el lado derecho. En la parte antetior parecen quedar restos de esta posible cincha subiendo hacia la grupa, pero todo ha sido limado casi al nivel de la superficie de la manta. En el vientre no aparece ningún rastro de la cincha.

El limado del reborde de la cobertura sobre la grupa, en su parte posterior, y la ausencia de este debajo del remache saliente, así como la falta de una clara representación de la cincha, nos hacen pensar en la posibilidad de que el caballo llevase un jinete, cuya pierna izquierda irfa sujeta al animal mediante el remache que sobresale. El limado de la parte superior del reborde de la montura sobre la grupa tendria como finalidad asentar mejor la figura del jinete sobre la yegua. Las piernas del jinete no permititían ver la cincha, por lo que su representación no fue planeada en el modelado de la pieza o fue considerada como algo sin importancia. 


\section{PARALELOS Y CRONOLOGIA}

Como ya hemos indicado, la pieza de bronce parece corresponder a un conjunto sobre plataforma que constaría, al menos, de jinete y caballo. Formalmente y por su tamaño, aunque no por su técnica, recuerda a los caballos de los llamados "carros votivos", como el de Mérida o Almorchón (7), si bien encontramos que el bronce de Cancho Roano ha sido modelado de manera más naturalista, consiguiendo un resultado más cercano a la realidad. De todas formas, salvando las diferencias artisticas, que no indican sino que los artifices de estas piezas no han sido los mismos, la pieza parece corresponder a este grupo de bronces de "carros votivos" extremeños que suelen representar escenas de caza. Tal vez en el conjunto de Cancho Roano falten, además del jinete, los demás elementos de la escena cinegética, así como las ruedas que soportan estas plataformas.

En cuanto al atalaje, debemos referirnos a las representaciones de caballos ibéricos. La montuta se puede clasificar dentro del grupo IV de la tipología de E. Cuadrado para los caballos del Cigarralejo (8). Se trata de una montura apuntada, sencilla y sin ningún tipo de decoración, sólo delimitada por un reborde de sección rectangular que sobresale ligeramente del cuerpo del animal.

Las viseras también aparecen representadas en algunas piezas fberas, como por ejemplo en el caballo con jinete de El Salobral (9) o en algún exvoto del santuario de Collado de los Jardines (10), pero se trata de elementos bastantes raros y no tenemos noticias de que haya aparecido ninguna visera en las excavaciones de nuestra península, lo cual puede ser debido a que estuvieran realizadas en materiales perecederos, aunque en Chipre se han encontrado algunos ejemplares realizados en bronce (11)

La ausencia de claros paralelos formales tanto dentro como fuera de la Península, asi como la técnica empleada, parecen avalar su adscripción a talleres peninsulares difíciles de ubicar en una época donde parece evidente la importancia que adquieren las zonas periféricas del territorio tartésico clásico. La presencia de crisoles y tortas de fundición en los alrededores del yacimiento (12), así como la originalidad formal que presentan los jarros y recipientes de manos en él hallados (13), hace suponer la existencia de algún taller de fundición cerca del Santuario, o bien, algún taller de reparación para el mantenimiento de los numerosos objetos de bronce en el hallados. Tampoco hay que olvidar la práctica de la refundición que debió desarrollarse, adaptando viejas piezas de bronce a las nuevas modas artisticas; en este sentido hay que hacer alusión al gran nứmero de objetos relacionados con el caballo aparecidos en Cancho Roano, pudiendo responder éste a esa idea sin duda relacionada con la importancia que debió tener el caballo en el Santuario (14)

(7) Blazquez, J.M. 1955. "Los carros votivos de Mérida y Almorchón" Zephyrus 6. Salamanca pp. 41 y ss. para el de Mérida, y pp. 58 y ss. para el de Almorçón. Una revisión de los mismos en Blazquez, J.M. 1976. "Bronces de la Mérida Prezromana” li Milenario de Merida Madrid.

(8) Cuadrado, E. 1950. "Excavaciones en el Santuario ibérico de El Cigarralejo (Mula, Murcia)" Informes y Memorias núm. 21. Madrid.

(9) Alvarez-Ossorio, F. 1941.. Catdloge de los extotos de bronce, ibtricos. M.A.N. Madid. Láminas. Lón. LXXIX, n. 606.

(10) Nicolini, G. 1969. Les brontes figurés des Sancruaires i6triques. P.U.F. Paris. pp. 56-59; P1. 1, 1-5.

(11) Karageorgis, V. 1967. Excavations in the necropolis of Salamis I. Nicosia. Tumba 3; fig. 111 y 22.

(12) Maluquer de Motes, J. 1983, p. 85.

(13) Celestino, S. e.p. "Nuevos jarzos tartésicos en el Sur de ia Peninsula Iberica" Madrider Mitteilungen 32 (1991).

(14) La presencia de objetos relacionados con los caballos es muy significativa en el Santuario, ver por ejemplo Maluçuer de Motes, J. 1981 figs. 10-12, 37-42 y 52; Maluquer de Motes J. 1983 figs. 9-25; Maluqquer de Motes, J. 1985. "Un arista extremaho de hace 2.500 afios ${ }^{\circ}$ Homenaje a Canovas Perini. Badajoz. 
Menos dificultad presenta su cronología si tenemos en cuenta que el yacimiento se fecha entre los siglos VI y V a. C. Sin embargo, parece probable que este tipo de piezas de prestigio debieron mantenerse en circulación durante bastante tiempo después del momento de su creación, no sólo por el ascendiente social que darían a su poseedor, sino también por la misma perdurabilidad del material con que están realizadas, en este caso el bronce. No obstante no hay elementos de juicio que permitan fecharla antes de finales del siglo VI a. C.

RESULTADO DE LOS ANALISIS (\% en peso)

Técrica: Espectrometría por fluorescencia de rayos-X

(Análisis de la superficie)

Espectrómetro KEVEX mod. 7000 del I.C.R.B.C. (Madrid)

\begin{tabular}{|c|c|c|c|c|c|c|c|c|c|c|c|}
\hline Yacimiento & \multicolumn{4}{|c|}{ Localidad } & \multicolumn{3}{|c|}{ Tipo de objeto } & \multicolumn{4}{|c|}{ Número de inventario } \\
\hline $\begin{array}{l}\text { Cancho Roano } \\
\text { Cancho Roano } \\
\text { Cancho Roano }\end{array}$ & $\begin{array}{l}\text { Zal } \\
\text { Zal } \\
\text { Zala }\end{array}$ & $\begin{array}{l}\text { Imea de } \\
\text { imea de } \\
\text { imea de }\end{array}$ & $\begin{array}{l}\text { la Seten } \\
\text { la Seren } \\
\text { la Seren }\end{array}$ & & $\begin{array}{l}\text { Est. Ca } \\
\text { Est. C } \\
\text { Est. }\end{array}$ & $\begin{array}{l}\text { ballo (c } \\
\text { Caballo } \\
\text { Caballo }\end{array}$ & $\begin{array}{l}\text { avo suj.) } \\
\text { (lomo) } \\
\text { (crin) }\end{array}$ & & & - & \\
\hline Número de análisis & $\mathrm{Fe}$ & $\mathrm{Ni}$ & $\mathrm{Cu}$ & $\mathrm{Zn}$ & As & $\mathrm{Ag}$ & $\mathrm{Sn}$ & $\mathrm{Sb}$ & $\mathrm{Au}$ & $\mathrm{Pb}$ & $\mathrm{Bi}$ \\
\hline $\begin{array}{l}\text { PA3174C } \\
\text { PA3174A } \\
\text { PA3174B }\end{array}$ & $\begin{array}{l}\overline{0.339} \\
0.224 \\
0.192\end{array}$ & $\begin{array}{l}0 . \overline{282} \\
0.119 \\
0.025\end{array}$ & $\begin{array}{l}87.67 \\
81.86 \\
80.78\end{array}$ & $\begin{array}{l}\text { nd } \\
\text { nd } \\
\text { nd }\end{array}$ & $\begin{array}{l}0.14 \overline{4} \\
\text { nd } \\
\text { nd }\end{array}$ & $\begin{array}{l}0.037 \\
0.061 \\
0.061\end{array}$ & $\begin{array}{l}10.25 \\
12.94 \\
12.61\end{array}$ & $\begin{array}{l}0.064 \\
0.129 \\
0.128\end{array}$ & - & $\begin{array}{l}1.047 \\
4.356 \\
6.217\end{array}$ & $\begin{array}{l}\text { nd } \\
\text { nd } \\
\text { nd }\end{array}$ \\
\hline
\end{tabular}

* Análisis realizado por el Dr. Salvador Rovira Llorens 


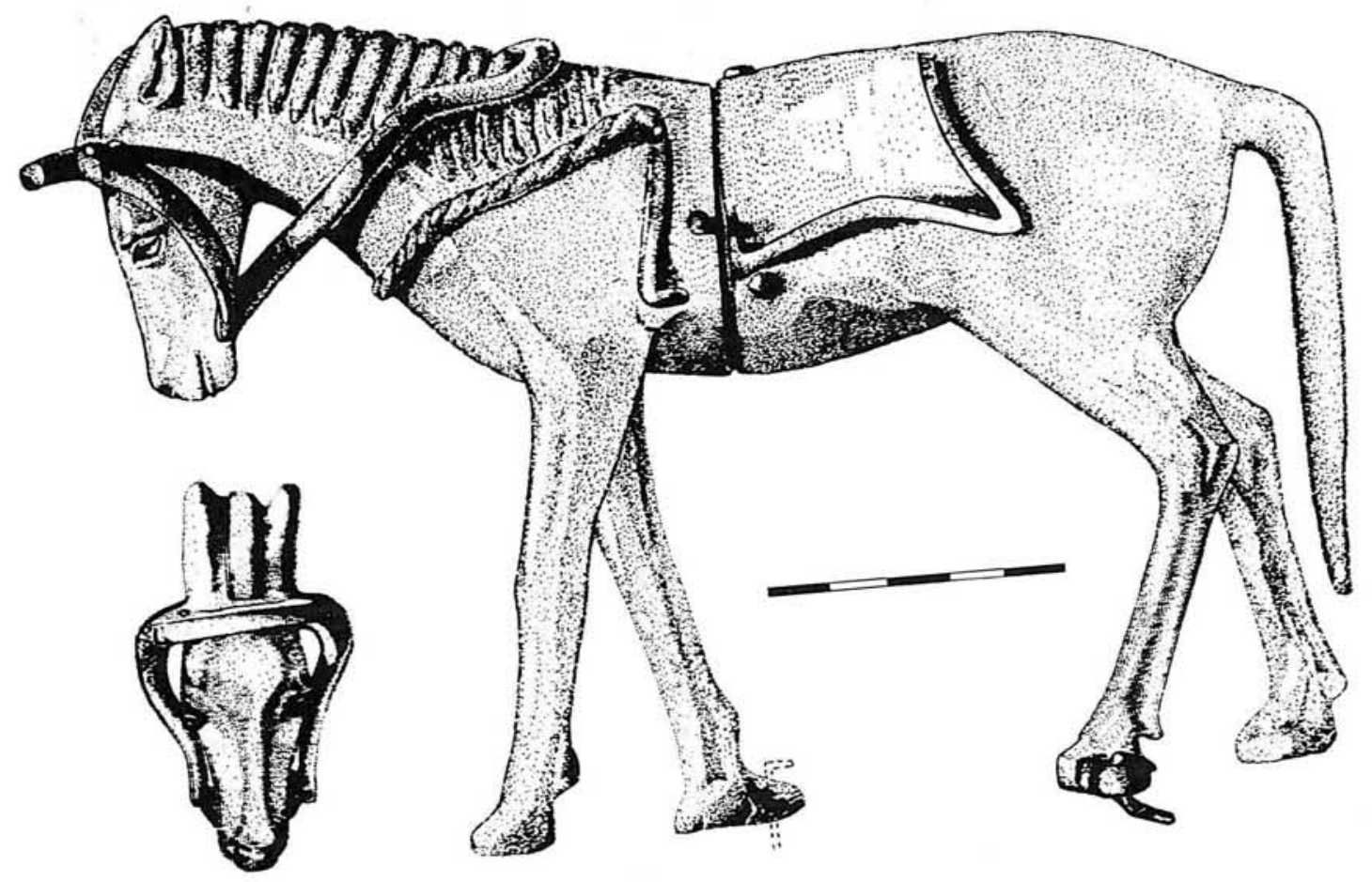

Figura 1 

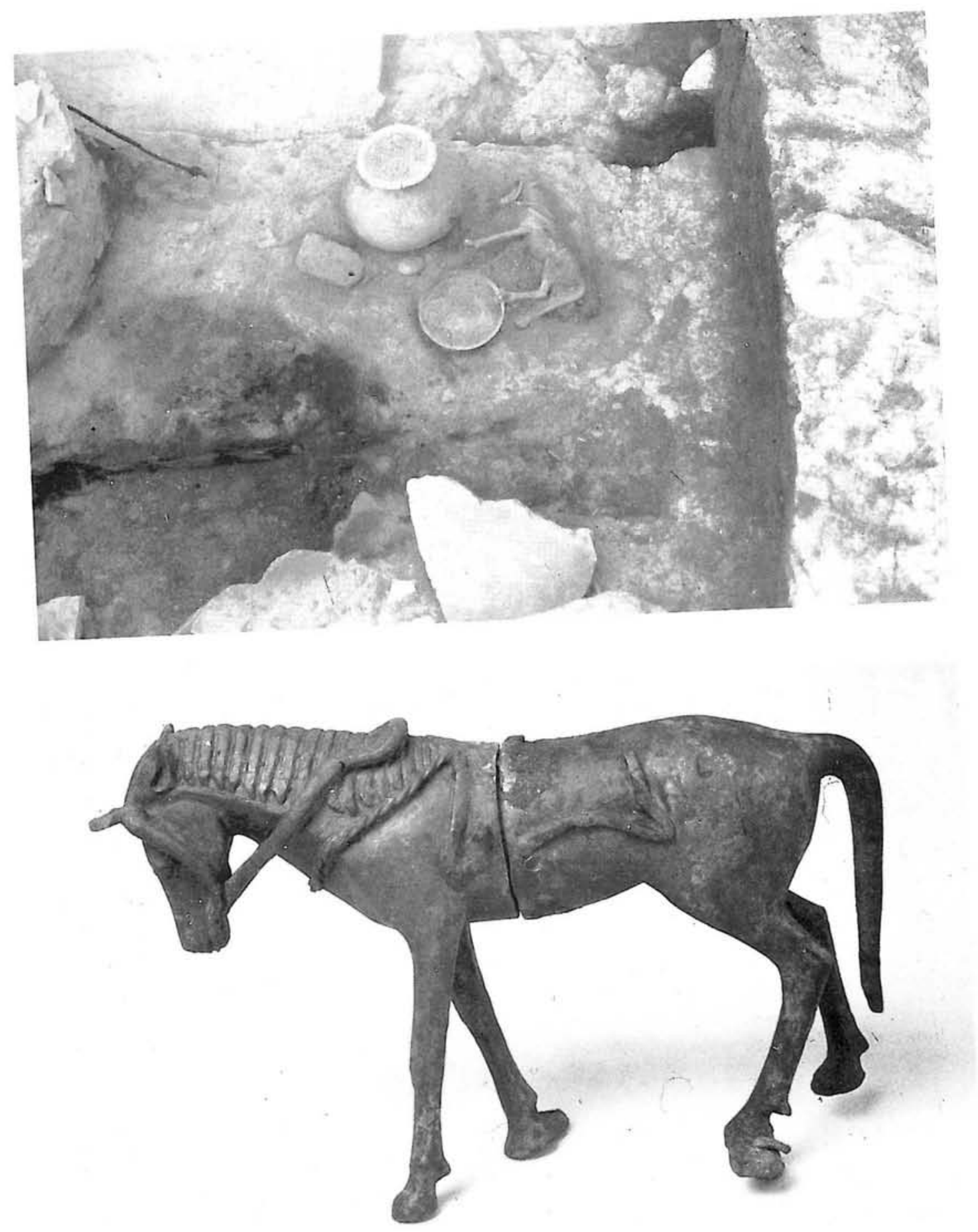

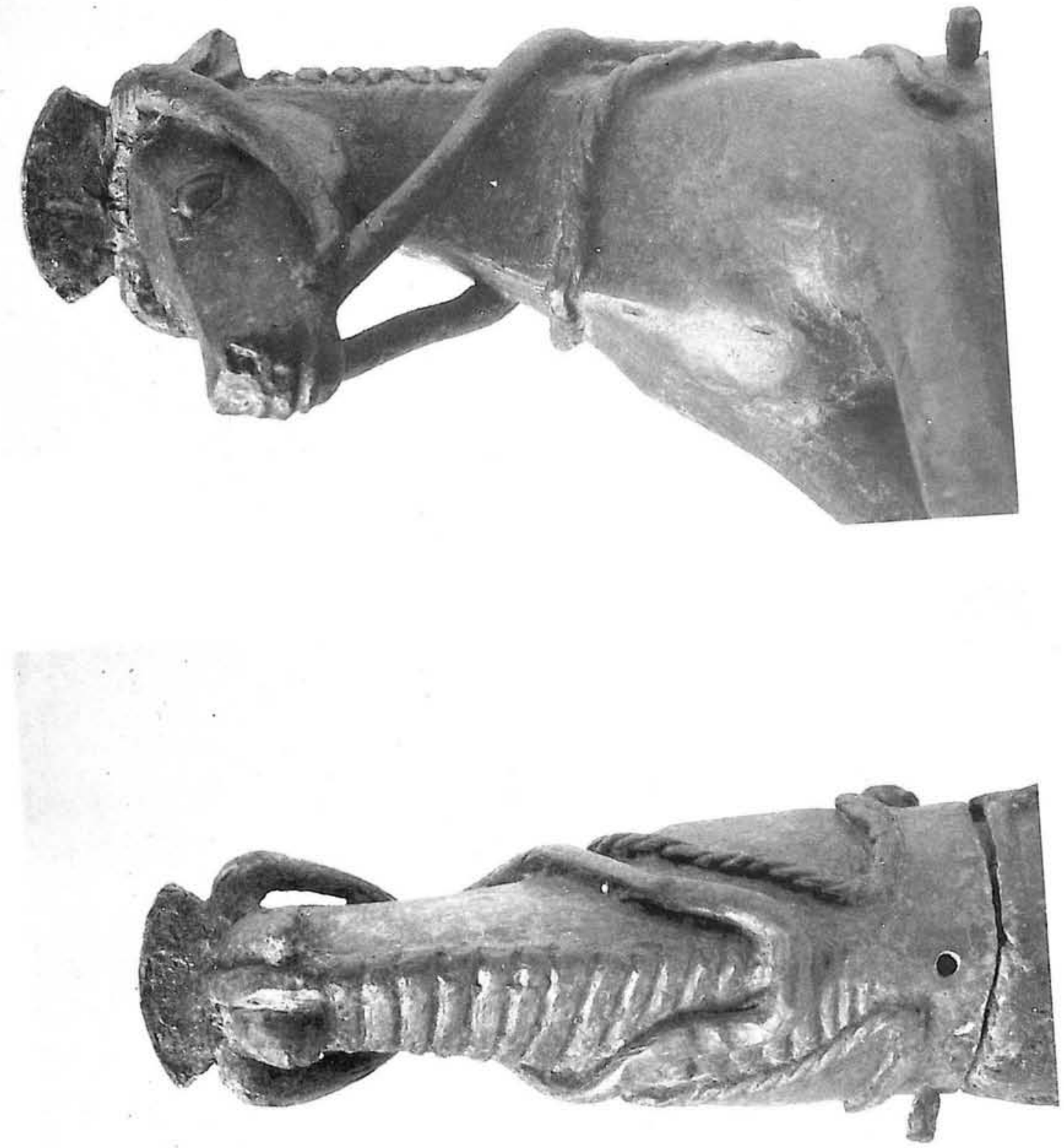


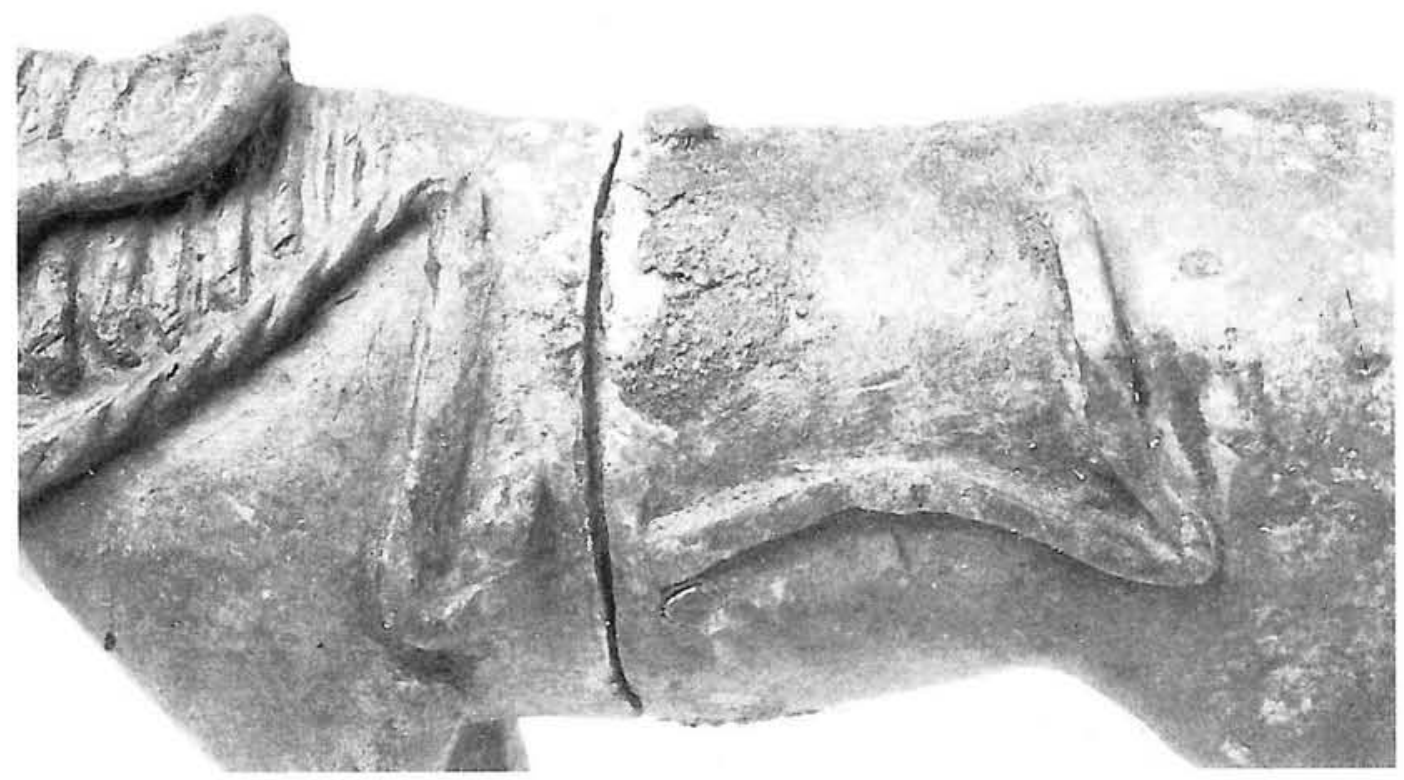

